\title{
Yellow nail syndrome following multiple orthopedic surgeries: a case report
}

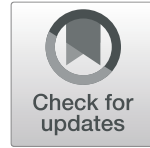

Hideya Itagaki* iD and Suzuki Katuhiko

\begin{abstract}
Background: Yellow nail syndrome is a rare condition associated with a triad of symptoms: yellow nails, lung lesions, and lymphedema. We report a case of yellow nail syndrome caused by titanium exposure from multiple artificial joint replacements.

Case presentation: A 78-year-old Asian woman presented to our outpatient department with chief complaints of cough, fever, and nausea. The patient was hospitalized for observation because of the presence of hypoxemia and bilateral pleural effusion. Her medical history included knee joint replacement and two spinal fusion surgeries. Her physical examination conducted following hospitalization revealed yellow nails on both hands and feet. This finding, combined with the observation of bilateral pleural effusion, raised suspicion for yellow nail syndrome. Blood analysis yielded negative results, as did the tests for sputum culture, interferon liberation, pleural effusion culture, and pleural effusion cytology. Pleural histopathological analysis and imaging yielded negative results. Considering the possibility of titanium exposure from artificial joints based on the patient's medical history, we examined a chest radiograph obtained before the second spinal fusion surgery; however, no pleural effusion was observed. Pleural effusion was observed, however, following the surgery. On the basis of these findings, the patient was diagnosed with yellow nail syndrome due to titanium exposure.

Conclusions: Clinicians should examine the nails of patients with unexplained pleural effusion. Moreover, they should inquire about titanium exposure when obtaining the patient's medical history.
\end{abstract}

Keywords: Yellow nail syndrome, Titanium, Spinal fusion, Pleural effusion

\section{Background}

Yellow nail syndrome (YNS) is a rare condition that was first identified by Samman et al. in 1964. YNS is characterized by a triad of symptoms: yellow nails, lung lesions, and lymphedema [1]. Although the condition's etiology remains unclear, YNS is associated with cancer and autoimmune diseases. YNS may also develop following dental treatment or titanium exposure from cardiac pacemakers or artificial joints [2]. Previously published case reports of YNS due to titanium exposure could not confirm the number of surgeries undergone by patients. Our patient had undergone joint replacement twice without the development of YNS; therefore, attributing titanium exposure as the cause of YNS following the third surgery was difficult because no disease onset was observed following the initial two surgeries. We report

\footnotetext{
* Correspondence: hideya.itagaki@gmail.com

Department of General Surgery, Honjoudaiichi Hospital, 110, Iwabuchishita, Yurihonnjou, Akita 015-8567, Japan
}

an interesting case of YNS caused by titanium exposure from multiple artificial joint replacement surgeries.

\section{Case presentation}

A 78-year-old Asian woman presented to our outpatient department with chief complaints of coughing and fever. Her cough had persisted for several weeks, and her fever had developed on the previous day. The patient's medical history included asthma and sinusitis. Although her sinusitis had been treated several years prior, she had not received treatment before hospitalization. The patient's surgical history included knee joint replacement and two spinal fusion surgeries; the second spinal fusion had been performed 3 months before the current consultation.

Physical examination revealed hypoxemia, and auscultation revealed bilateral chest crackles with no sign of heart failure. Bilateral pleural effusion was detected on a chest radiograph (Fig. 1). The patient developed

(c) The Author(s). 2019 Open Access This article is distributed under the terms of the Creative Commons Attribution 4.0 International License (http://creativecommons.org/licenses/by/4.0/), which permits unrestricted use, distribution, and 


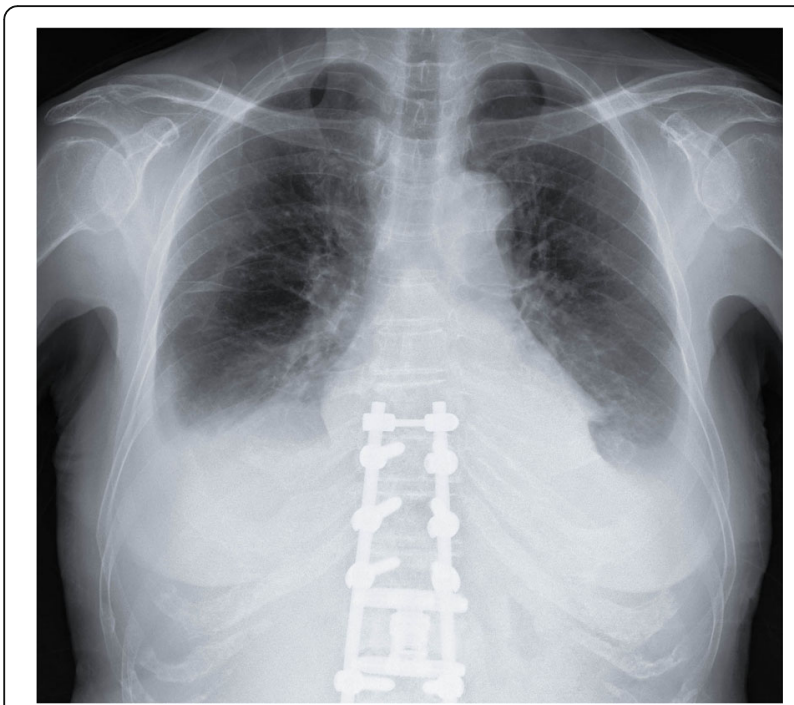

Fig. 1 Bilateral pleural effusion was detected on a chest radiograph at the time of hospitalization

yellowing of her fingernails and toenails following hospitalization (Figs. 2 and 3). This finding, combined with the patient's pleural effusion and sinusitis, led to suspicion for YNS. Bilateral dorsum pedis lymphedema was confirmed during hospitalization. Blood analysis revealed a slight increase in inflammation. However, the patient's test results for rheumatoid factor and anticyclic citrullinated peptide antibody were negative. Her thyroid parameters and levels of soluble interleukin-2 receptor were normal. Her sputum culture and interferon$\gamma$ release assay results were negative. The result of her bacterial culture of pleural effusion was negative.
Computed tomography failed to confirm the presence of a malignant tumor. A chest radiograph (Fig. 4) obtained prior to the second spinal fusion procedure showed no pleural effusion. However, pleural effusion appeared 1 month after the second surgery. On the basis of these findings, the patient was diagnosed with YNS due to titanium exposure.

After diagnosis, vitamin $\mathrm{E}$ was administered for more than 1 year. After a half-year of vitamin $E$ administration, improvement in the thickness of the nails on the patient's hands was observed (Fig. 5), but no effect was seen for the pleural effusion. Pleural effusion also failed to respond to pleurodesis. Pleural effusion drainage was therefore performed regularly. Currently, the patient visits our clinic every 1-2 months and undergoes chest radiography. Pleural drainage is performed if there is an increase in pleural fluid.

\section{Discussion}

YNS is a rare condition with an estimated incidence of 1 in 1,000,000 [1,3]. It typically affects individuals aged between 50 and 60 years, with both genders being similarly affected [4]. The etiology of YNS remains unclear, but it may involve an acquired dysfunction of the lymphatic circulation.

Nail abnormalities are found in $90-100 \%$ of patients with YNS [3], and they include yellow discoloration, nail-plate thickening, detachment, delayed growth, transverse ridges, excessive curvature, and pains. YNS generally affects all the nails of both hands and feet. Respiratory lesions are observed in $50-70 \%$ of patients with YNS, with associated chronic cough, pleural effusion, bronchiectasis, recurrent pneumonia, and chronic

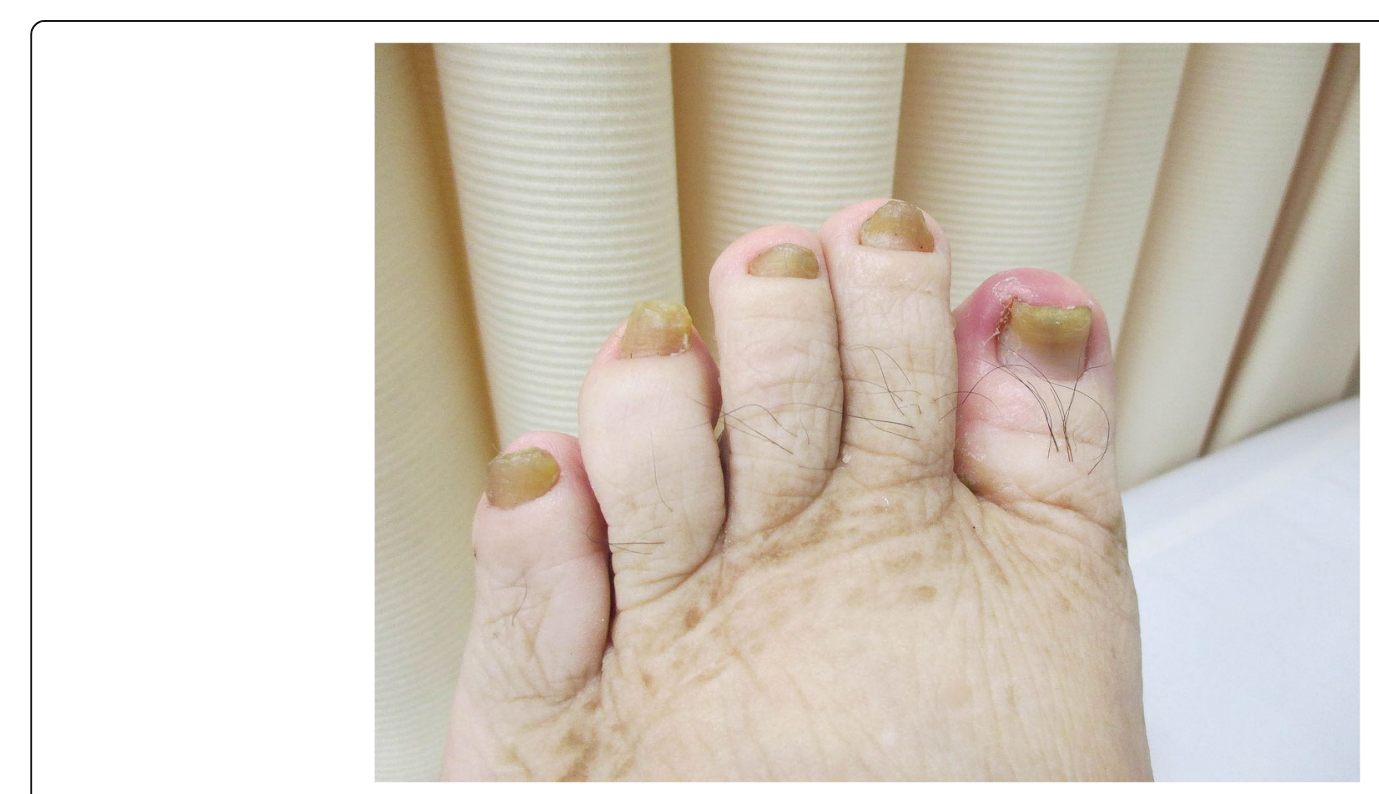

Fig. 2 Yellow toenails 


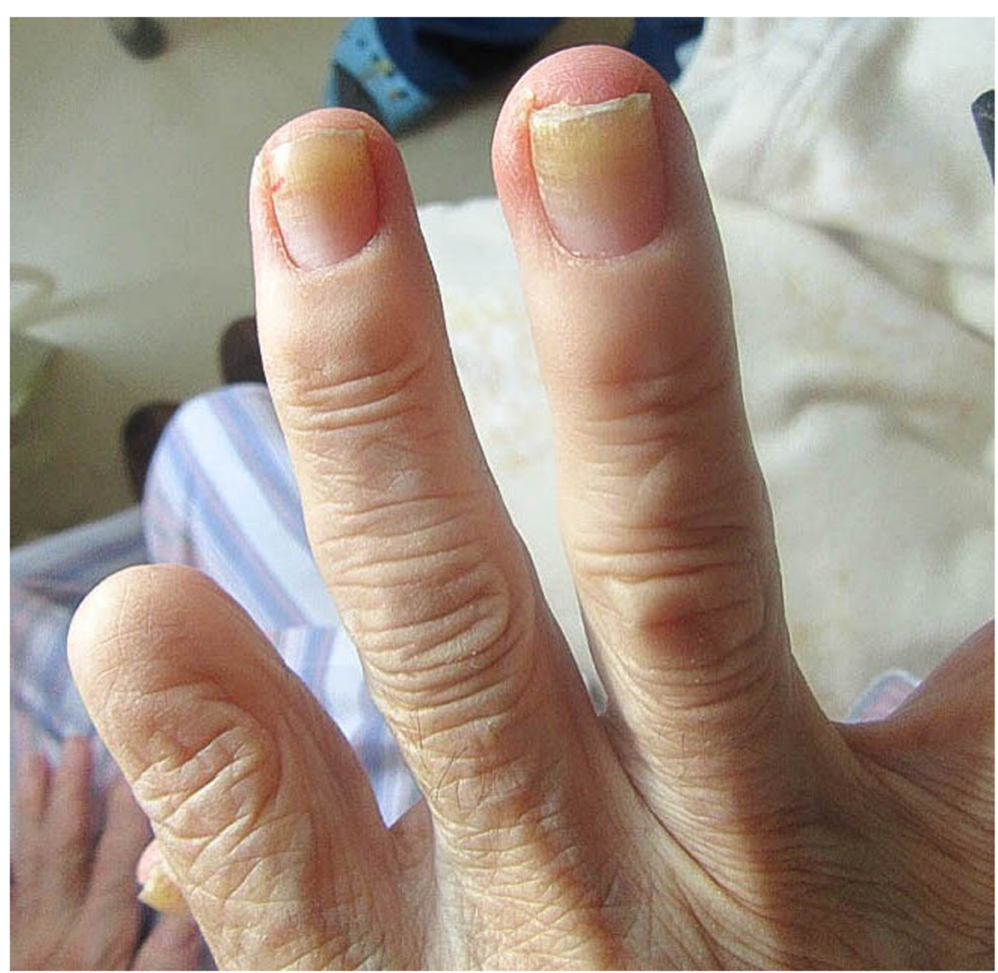

Fig. 3 Yellow fingernails. The right index finger distal interphalangeal joint (DIP) and beyond are lost due to trauma

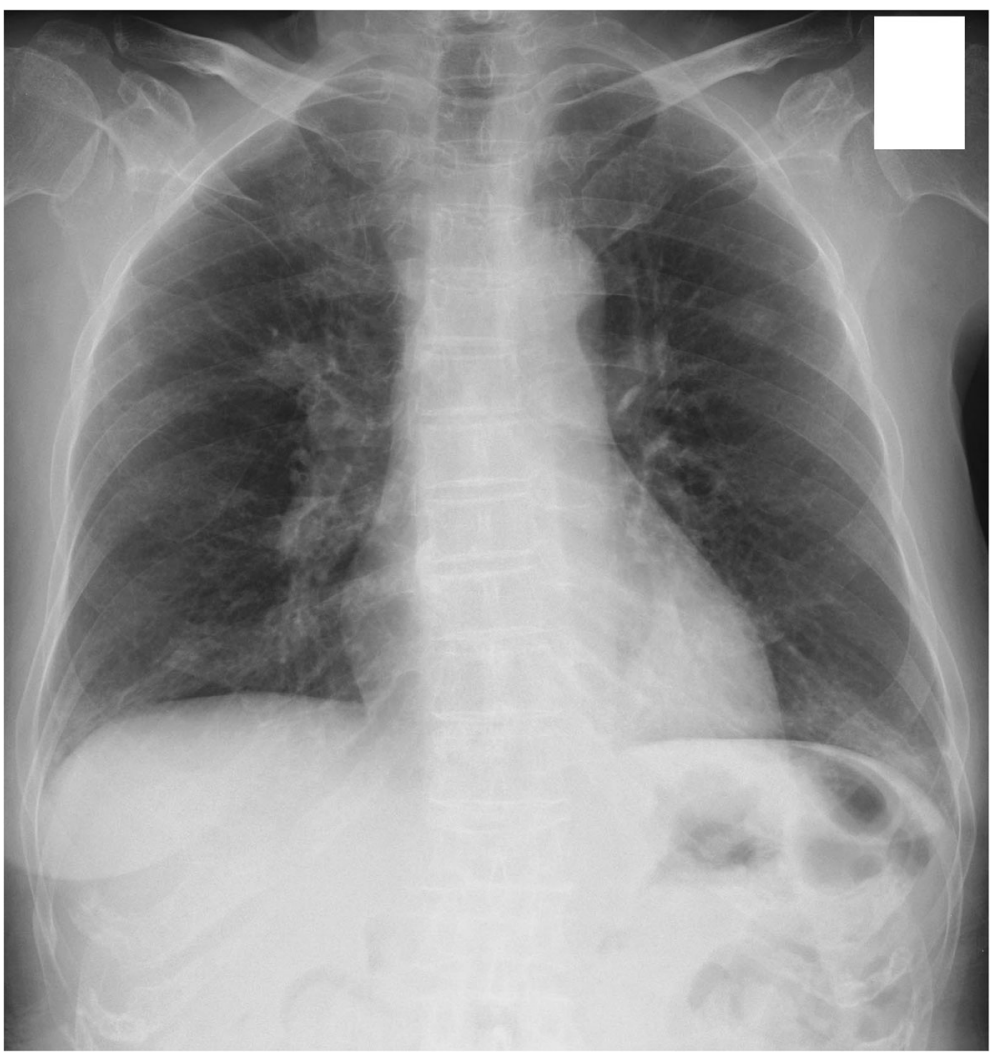

Fig. 4 Chest radiograph obtained prior to the second spinal fusion showing no pleural effusion 


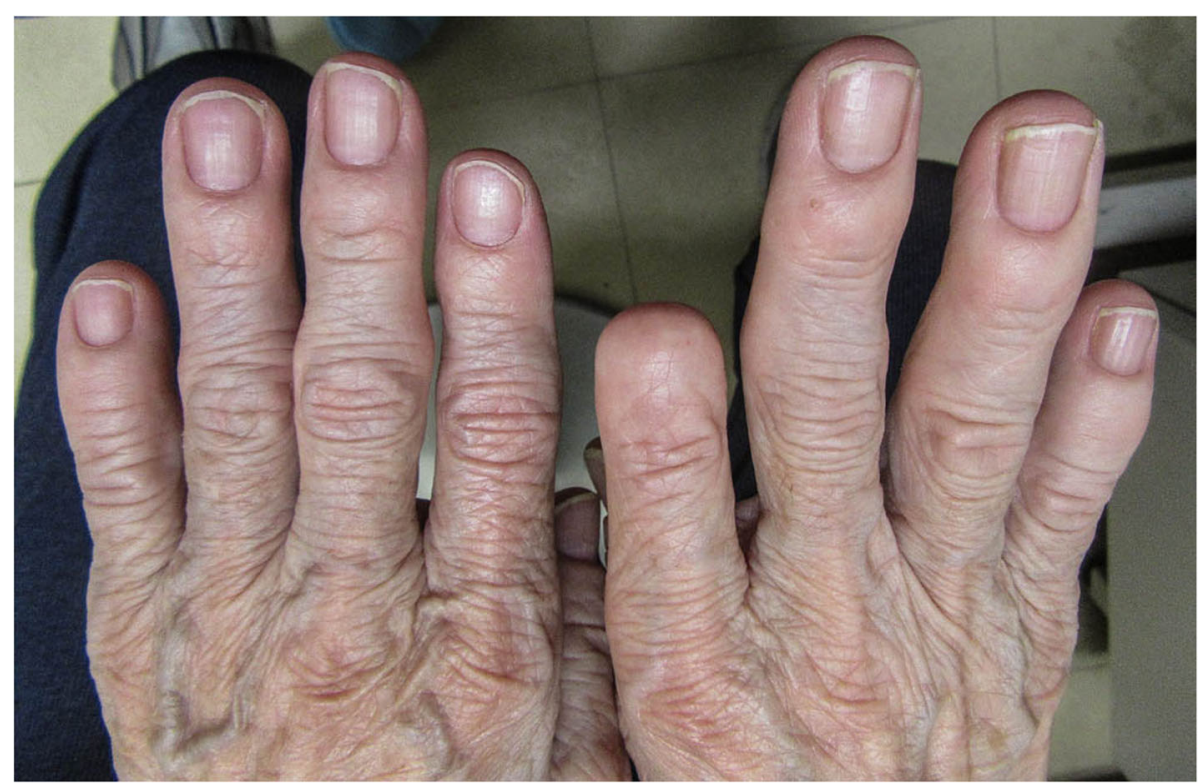

Fig. 5 Improvement in the thickness of the nails on the hands

sinusitis. The most common YNS symptom is chronic cough, found in $56 \%$ of patients with YNS [4]. Pleural effusion is bilateral in $68.3 \%$ patients and exudative in $94.7 \%$, with lymphocytes predominant in the exudate (96\%) [5]. Lymphedema is observed in $30-80 \%$ of patients with YNS [3]; it appears in both lower extremities, usually below the knee. Increased lymphedema is considered to be indicative of the excessive accumulation of lymphocytes and fiber formation that is caused by the stimulation of fibroblasts and adipocytes.

The presence of two symptoms from among the triad of yellow nails, lung lesions, and lymphedema is sufficient for a diagnosis of YNS because the three characteristic symptoms do not typically appear simultaneously. All three YNS symptoms are present in $27-76 \%$ of cases $[6,7]$. Our patient's case of YNS was diagnosed on the basis of the presence of yellow nails and respiratory lesions (chronic cough, bilateral pleural effusion, and sinusitis) as well as lymphedema noted on both feet during hospitalization. The patient thus presented with all three symptoms over time. Pleural effusion was bilateral and exudative with lymphocyte-predominant white blood cells; this finding was consistent with YNS.

YNS has been associated with various diseases, including cancer, autoimmune dysfunction, immunodeficiency, nephrotic syndrome, hyperthyroidism, hypothyroidism, rheumatoid arthritis, and tuberculosis [3]. Moreover, titanium exposure has also been implicated [8]. Berglund et al. [8] used energy-dispersive X-ray fluorescence to analyze nail samples from 30 patients with at least one YNS symptom, and the results showed that high levels of titanium $(1.1-170 \mu \mathrm{g} / \mathrm{g}$ nail) were present. The authors also reported that gold or amalgam (alloys of mercury and other metals used as dental filling material) could induce galvanic corrosion of titanium, resulting in the release of titanium ions. The removal of gold teeth from four patients wearing titanium implants resulted in the complete resolution of symptoms. Titanium removal is therefore recommended. However, for cases in which titanium removal is difficult, further research is necessary to determine an alternative approach to treatment.

Previous studies have demonstrated that vitamin E supplementation [9], antifungal agents [10], and clarithromycin $[11,12]$ are effective in the treatment of YNS. In our patient's case, oral administration of vitamin $\mathrm{E}$ was recommended as treatment because the removal of titanium was difficult. Vitamin E may be administered locally (topical vitamin E) or orally (oral vitamin E). Local administration prevents the deposition of lipofuscin, which causes nail yellowing, and is therefore considered to be an effective treatment for nail symptoms [13]. Oral administration also reportedly prevents the deposition of lipofuscin production that is possibly responsible for nail yellowing [14]. Symptoms have reportedly been resolved with oral administration of $800 \mathrm{mg}$ of vitamin $\mathrm{E}$ as a first dose, followed by $400 \mathrm{mg}$ every $24 \mathrm{~h}$ [9]. Other researchers have reported an improvement in symptoms with the simultaneous oral administration of vitamin $\mathrm{E}$ and antifungal drugs $[10,15]$. However, using antifungal drugs alone remains controversial. Tosti et al. administered itraconazole to patients with YNS but did not report on its efficacy [16]. In our patient's case, although oral vitamin E improved the patient's nail color, it had no effect on pleural effusion. Therefore, we proceeded 
with pleurodesis. Surgical intervention is an effective treatment for pleural effusion. According to Valdés et $a l$., the effective rates of pleurodesis, decortication/pleurectomy, and pleural-peritoneal shunt for pleural effusion in patients with YNS were $81.8 \%, 88.9 \%$, and $66.7 \%$, respectively [5]. For pleurodesis, talc [17] and OK-432 [18] are generally used as pleurodesis adhesion materials. In our patient's case, OK-432 was used for pleurodesis. However, pleurodesis failed to improve the pleural effusion; therefore, we regularly performed pleural effusion drainage.

\section{Conclusions}

YNS, which may be caused by titanium exposure, is a rare complication of multiple joint replacement surgeries. A patient's nails should be examined in the case of an unexplained pleural effusion, and the surgical history, particularly the number of operations the patient has undergone, should be evaluated. As the population ages and the number of patients with artificial joints increases, the incidence of YNS may also increase. Surgical intervention is effective in the treatment of pleural effusion in patients with YNS.

\section{Abbreviation}

YNS: Yellow nail syndrome

\section{Acknowledgements}

The authors thank Enago (www.enago.jp) for English-language review.

\section{Authors' contributions}

$\mathrm{HI}$ treated the patient, interpreted the data, and contributed to preparing the manuscript. SK analyzed the manuscript. Both authors read and approved the final manuscript.

\section{Funding}

The authors declare that no funding was received for this study.

\section{Availability of data and materials}

Data sharing is not applicable to this article, because no datasets were generated or analyzed during the current study.

\section{Ethics approval and consent to participate}

This study was approved by the Honjou Daiichi Hospital Ethics Committee.

\section{Consent for publication}

Written informed consent was obtained from the patient for publication of this case report and any accompanying images. A copy of the written consent is available for review by the Editor-in-Chief of this journal.

\section{Competing interests}

The authors declare that they have no competing interests.

Received: 18 March 2019 Accepted: 29 May 2019

Published online: 01 July 2019

\section{References}

1. Samman PD, White WF. The "yellow nail" syndrome. Br J Dermatol. 1964;76: 153-7.

2. Suzuki T, Tokuda Y, Kobayashi H. The development of yellow nail syndrome after the implantation of a permanent cardiac pacemaker. Intern Med. 2017; 56(19):2667-9.

3. Vignes $S$, Baran R. Yellow nail syndrome: a review. Orphanet J Rare Dis. 2017;12(1):42.
4. Maldonado F, Tazelaar HD, Wang CW, Ryu JH. Yellow nail syndrome: analysis of 41 consecutive patients. Chest. 2008;134(2):375-81.

5. Valdés L, Huggins JT, Gude F, et al. Characteristics of patients with yellow nail syndrome and pleural effusion. Respirology. 2014;19(7):985-92.

6. Piraccini BM, Urciuoli B, Starace M, Tosti A, Balestri R. Yellow nail syndrome: clinical experience in a series of 21 patients. J Dtsch Dermatol Ges. 2014; 12(2):131-7

7. Pavlidakey GP, Hashimoto K, Blum D. Yellow nail syndrome. J Am Acad Dermatol. 1984:11(3):509-12.

8. Berglund F, Carlmark B. Titanium, sinusitis, and the yellow nail syndrome. Biol Trace Elem Res. 2011:143(1):1-7.

9. Ayres S Jr, Mihan R. Yellow nail syndrome: response to vitamin E. Arch Dermatol. 1973;108(2):267-8.

10. Luyten C, Andre J, Walraevens C, De Doncker P. Yellow nail syndrome and onychomycosis: experience with itraconazole pulse therapy combined with vitamin E. Dermatology. 1996;192(4):406-8.

11. Suzuki M, Yoshizawa A, Sugiyama $H$, et al. A case of yellow nail syndrome with dramatically improved nail discoloration by oral clarithromycin. Case Rep Dermatol. 2011;3(3):251-8.

12. Matsubayashi S, Suzuki M, Suzuki T, et al. Effectiveness of clarithromycin in patients with yellow nail syndrome. BMC Pulm Med. 2018;18:138.

13. Williams HC, Buffham R, du Vivier A. Successful use of topical vitamin E solution in the treatment of nail changes in yellow nail syndrome. Arch Dermatol. 1991:127(7):1023-8.

14. Norton L. Further observations on the yellow nail syndrome with therapeutic effects of oral alpha-tocopherol. Cutis. 1985;36(6):457-62.

15. Baran R, Thomas L. Combination of fluconazole and alpha-tocopherol in the treatment of yellow nail syndrome. J Drugs Dermatol. 2009;8(3):276-8.

16. Tosti A, Piraccini BM, lorizzo M. Systemic itraconazole in the yellow nail syndrome. Br J Dermatol. 2002;146(6):1064-7.

17. Balmforth D, Bille A, Okiror L, Harrsion-Phipps K, Routledge T. Recurrent pleural effusion in yellow nail syndrome successfully treated with videoassisted thoracic surgery: comparison of two surgical strategies in two cases. Gen Thorac Cardiovasc Surg. 2013;61(4):231-3.

18. Yamagishi $T$, Hatanaka $\mathrm{N}$, Kamemura $\mathrm{H}$, et al. Idiopathic yellow nail syndrome successfully treated with OK-432. Intern Med. 2007;46(14):1127-

\section{Publisher's Note}

Springer Nature remains neutral with regard to jurisdictional claims in published maps and institutional affiliations.
Ready to submit your research? Choose BMC and benefit from:
- fast, convenient online submission
- thorough peer review by experienced researchers in your field
- rapid publication on acceptance
- support for research data, including large and complex data types
- gold Open Access which fosters wider collaboration and increased citations
- maximum visibility for your research: over $100 \mathrm{M}$ website views per year
At $\mathrm{BMC}$, research is always in progress.
Learn more biomedcentral.com/submissions 